\title{
A Techniques to Downgrade Objective Function in Parallel Robot Kinematics Problem
}

\author{
Trang Thanh Trung*, Li Wei Guang*, Pham Thanh Long** \\ * School of Mechanical and Automotive Engineering, South China University of Technology, China \\ ** Faculty of Mechanical Engineering, Thai Nguyen University of Technology, Vietnam
}

\section{Article Info \\ Article history: \\ Received Apr 6, 2015 \\ Revised Aug 21, 2015 \\ Accepted Aug 29, 2015 \\ Keyword: \\ Equivalent structure Kinematics model Kinematics problem Objective functions Parallel robot}

\begin{abstract}
Quadratic functions and quaternary are preferable forms solving parallel robot kinematics problems. In order to simplify and to use only one method to solve all forms of the objective functions, this article introduces prevalent techniques to downgrade mathematical model of the objective functions from quaternary function to quadratic function in parallel robot kinematics problem. By using equivalent alternative kinematic structure and additional mathematical constraints, we will change from the study of parallel robot kinematics problem with the form of quaternary objective function as the original configuration to equivalent alternative configuration in the form of quadratic objective function. The parallel robot kinematics problem based on alternative configuration with quadratic objective function is not only simpler but also helps to quickly identify the mathematical relationships in the joint space and work space of parallel robot. Then, more accurate control solution of parallel robot kinematics problem can be identified. Moreover, by using techniques in this study, all forms of objective functions of parallel robot of any structure can be easily solved. The result from numerical simulation has been used to prove the presented approaches.
\end{abstract}

Copyright $(2015$ Institute of Advanced Engineering and Science. All rights reserved.

\section{Corresponding Author:}

Trang Thanh Trung,

School of Mechanical and Automotive Engineering,

South China University of Technology,

Wushan road, Tianhe District, Guangzhou, Guang Dong, China.

Email: trangthanhtrung@gmail.com

\section{INTRODUCTION}

Day by day, the applications of the parallel manipulator in various field is become apparent and with a rapid rate utilized in precise manufacturing, medical science and in space exploration equipment [1]. The parallel manipulator can be defined as a closed-chain mechanism which has two platforms (base and moving platform), connected together by at least two independent kinematic chains [2]. The main advantages of parallel manipulator over serial robot include higher stiffness, better accuracy, larger load capacity, higher velocity and acceleration; higher throughput and non-accumulation of joint errors [3, 4]. Despite of its advantages, the parallel manipulator poses some challenges regarding to the limited workspace, singularities within the workspace and, finally, their kinematics model resolution [5]. Since the kinematics of parallel robot problem often contains the solving of the non-linear transcendental equations, it is not always possible to obtain a closed form solution [6]. Therefore, the selection of an efficient mathematical model is very crucial to simplify the kinematics problems in parallel robots [3].

Kinematic models of parallel robots can be built up by using geometric methods or the Denavit Hartenberg (DH) rule. However, to avoid the complicated factors of applying the DH rule, a geometric method is commonly used for modeling by changing the screw joints and spherical joints to the joints of 5 type [7]. By using this method, kinematic models are established, and investigating the kinematic problems of parallel robots can be done by applying optimized algorithm [8]. The obtained mathematical model for 
revolute joints and prismatic joints will be quadratic functions and quaternary functions respectively [9]. For the applications of the parallel structures having limb configurations of prismatic joints $(\mathrm{P})$ is active joint example RPR or SPS types, the optimization algorithm is no longer appropriate when it encounters quaternary objective functions. In addition, if the quaternary objective functions are kept, it is necessary to use the technique from [10]. Because of desiring continue to use optimized algorithm to solve kinematic problems of parallel robots. This paper presents an alternative configuration to downgrade the order of complicated objective functions to the simpler form. The new technique of changing variables introduced in this paper is suitable for parallel robots with different structure. Therefore, the objective desire of using a single method to study the kinematics for all kinds of parallel robots will gradually become real.

\section{THE FORM OF THE EQUATION WHEN TRANSFORMING PARALLEL ROBOT KINEMATICS PROBLEM INTO THE OPTIMIZATION PROBLEM}

Suppose that $\vec{P}\left(p_{x}, p_{y}, p_{z}, \alpha, \beta, \gamma\right)$ is the vector representing position and orientation of end effector of the robot. The aim of kinematic control is to achieve high accuracy of position and orientation of the end effector. Therefore, it is necessary to determine the joint variable $\theta_{i}$ so that the error of position and orientation is smallest while satisfying the conditions of structural constraints.

$\theta=\left(\theta_{1}, \theta_{2}, \ldots \theta_{n}\right):$ is the vector of joint variables.

Joint space $\mathrm{D}$ determines the domain of the following joint variables:

$\left\{\begin{array}{l}a_{1} \leq \theta_{1} \leq b_{1} \\ a_{2} \leq \theta_{2} \leq b_{2} \\ \vdots \\ a_{n} \leq \theta_{n} \leq b_{n}\end{array}\right.$

where: $a_{i}$ and $b_{i}$ are constraints of joint variables $\theta_{i}$.

$T=f(\theta)$ : function describing the deviation of position and orientation of the end effector.

The problem determining the value of the joint variables is written as follows:

$\left\{\begin{array}{l}T=f\left(\theta_{1}, \theta_{2}, \ldots \theta_{n}\right) \rightarrow \text { minimize } \\ a_{i} \leq \theta_{i} \leq b_{i} \\ \theta_{i} \in D ; i=1 \div n\end{array}\right.$

This is the equation of parallel robot kinematics problem when transformed into problem optimizing $n$ unknown nonlinear function, with linear constraints.

\section{ASSOCIATED EQUATION OF PARALLEL ROBOT WHEN DRIVEN BY REVOLUTE} JOINTS OR PRISMATIC JOINT

3.1. Associated equation when driven by revolute joints

In parallel robot 3RRR driven by revolute joints as Figure 1.

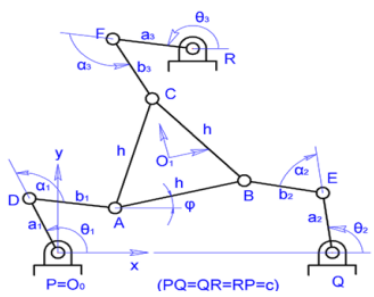

Figure 1. Parallel robot with 3RRR structur 
Because the robot has symmetrical structure when taking into consider the closed loop vector equation passing through the points $O_{0} \equiv P, D, A, O_{1}$ of the $1^{\text {st }}$ limb:

$$
\overrightarrow{O_{0} O_{1}}=\overrightarrow{O_{0} D}+\overrightarrow{D A}+\overrightarrow{A O_{1}}
$$

The detailed equation has form:

$$
\begin{aligned}
& \left|\begin{array}{l}
x_{01} \\
y_{01}
\end{array}\right|=\left|\begin{array}{l}
a_{1} \cdot \cos \left(\theta_{1}\right) \\
a_{1} \cdot \sin \left(\theta_{1}\right)
\end{array}\right|+\left|\begin{array}{l}
b_{1} \cdot \cos \left(\theta_{1}+\alpha_{1}\right) \\
b_{1} \cdot \sin \left(\theta_{1}+\alpha_{1}\right)
\end{array}\right|+\left|\begin{array}{l}
\frac{h \sqrt{3}}{3} \cdot \cos (\varphi+30) \\
\frac{h \sqrt{3}}{3} \cdot \sin (\varphi+30)
\end{array}\right| \\
& \Leftrightarrow\left\{\begin{array}{l}
x_{01}=a_{1} \cdot \cos \left(\theta_{1}\right)+b_{1} \cdot \cos \left(\theta_{1}+\alpha_{1}\right)+\frac{h \sqrt{3}}{3} \cdot \cos (\varphi+30) \\
y_{01}=a_{1} \cdot \sin \left(\theta_{1}\right)+b_{1} \cdot \sin \left(\theta_{1}+\alpha_{1}\right)+\frac{h \sqrt{3}}{3} \cdot \sin (\varphi+30)
\end{array}\right.
\end{aligned}
$$

When kinematic problem is structured based on (2), value of joint variables $\theta_{1}, \alpha_{1}$ is determined so that position error $x_{O 1}, y_{O 1}$ and orientation error $\varphi$ of the end effector reach minimum value. Therefore, objective function $\mathrm{T}$ describing deviation of position and orientation of the end effector is determined as follows:

$$
\begin{aligned}
& T=\left[x_{01}-\left(a_{1} \cdot \cos \left(\theta_{1}\right)+b_{1} \cdot \cos \left(\theta_{1}+\alpha_{1}\right)+\frac{h \sqrt{3}}{3} \cdot \cos (\varphi+30)\right)\right]^{2}+ \\
& {\left[y_{01}-\left(a_{1} \cdot \sin \left(\theta_{1}\right)+b_{1} \cdot \sin \left(\theta_{1}+\alpha_{1}\right)+\frac{h \sqrt{3}}{3} \cdot \sin (\varphi+30)\right)\right]^{2} \Rightarrow \text { minimize }}
\end{aligned}
$$

Obviously, (5) is always $\geq 0$, thus minimize value achieved is 0 equivalent to (4) satisfied. Function (5) has its own name called Rosenbrock-Banana function [11], the most appropriate algorithm to solve this functional is Generalized Reduced Gradient (GRG) algorithm [9, 12].

\subsection{Associated equation when driven by prismatic joints}

Consider detailed diagram of one limb $\mathrm{OA}_{3} \mathrm{~B}_{3} \mathrm{P}$ of parallel robot with $3 \mathrm{R} \underline{\mathrm{PS}}$ structure (Figure 2).

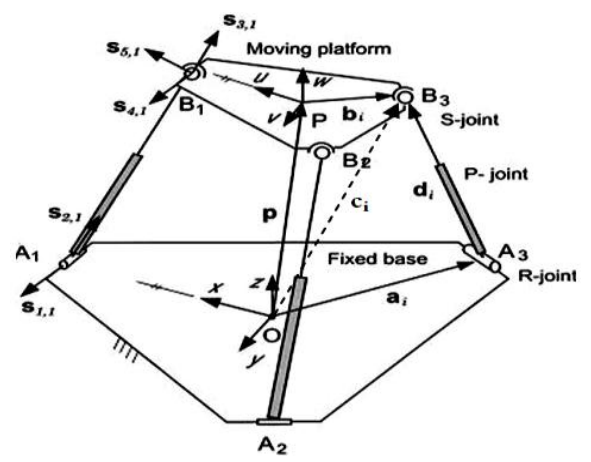

Figure 2. Parallel robot with 3RPS structure

Associated vector equation has the following form:

$$
\overrightarrow{d_{i}}=-\overrightarrow{a_{i}}+\vec{P}+R_{R P Y} \cdot \overrightarrow{b_{i}}
$$


The length of $i^{t h}$ limb that is the distance between two ends of vector $\vec{c}_{i}$ and $\vec{a}_{i}$ is determined as follows:

$$
d_{i}=\sqrt{\left(c_{i x}-a_{i x}\right)^{2}+\left(c_{i y}-a_{i y}\right)^{2}+\left(c_{i z}-a_{i z}\right)^{2}}=\sqrt{\left(\overline{c_{i}}-\overline{a_{i}}\right)\left(\overline{c_{i}}-\overline{a_{i}}\right)}
$$

When the objective function is structured based on (2), similar to section A, objective function is in the form of quaternary function due to the presence of prismatic joint variables $d_{i}$ :

$$
T=\sum_{i=1}^{n}\left[\left(c_{i x}-a_{i x}\right)^{2}+\left(c_{i y}-a_{i y}\right)^{2}+\left(c_{i z}-a_{i z}\right)^{2}-d_{i}^{2}\right]^{2} \Rightarrow \text { minimize }
$$

\section{EQUIVALENT ALTERNATIVE KINEMATIC MODEL}

From section 3, it can be seen that for the parallel robot driven by the prismatic joints, the objective function of the parallel robot kinematics problem always is in quaternary form, thus GRG algorithm is no longer appropriate to solve this form of function. To solve objective function containing fourth order variables, there are some appropriate methods such as Newton Raphson, Hook-Jeeves, Fletcher Powell [10] . In order to simplify and to use only one method - the GRG algorithm to solve the quaternary objective function in parallel robot kinematics problem. This article presents a method to downgrade the objective function based on equivalent alternative kinematic configuration. The down gradation of objective function is not merely a change of variable in mathematical models, but also requires appropriate kinematic structure between the two configurations.

\subsection{Methods to determine equivalent alternative configuration}

The determination of equivalent alternative configuration should be based on the structure of the robot to switch to an equivalent kinematic structure driven by the revolute joint instead of structure driven by the prismatic joint to avoid quaternary function as mentioned above.

\subsubsection{In case of using one revolute joint to replace one prismatic joint}

In case of the movement of the prismatic joint axis changes as parallel robot 3RPS, 6SP $\underline{P}$, equivalent alternative configuration proposed for this form of structure show in Figure 3.

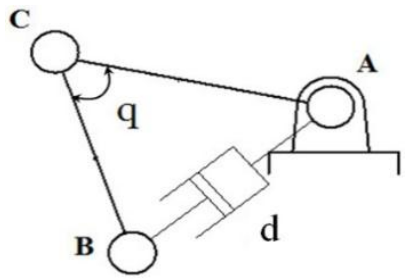

Figure 3. Equivalent structure when prismatic joints are replaced by revolute joints

The alternative configuration is used to create mathematical models having the form of quadratic objective function. It is required to retain the robot's actuators; only the drive part is replaced to get a structure with similar kinematic functions.

In the alternative configuration requires calculation of factors such as the direction of joint axis, activity limitations of revolute joints in specific relation with movement limitations of prismatic joints in the original configuration so that shape and volume of the workspace of the two structures is the same.

In Figure 3, AB limb originally is a prismatic joint (full association of the limb is S $\underline{P S}$ ) with control variable is the distance between two points $\mathrm{A}, \mathrm{B}$, and equal to $d$. AB limb can be replaced by ACB limb with SRS configuration, the degrees of freedom of the structure remains unchanged. In order to keep the volume and workspace of the robot constant it is necessary to convert keep overhead equivalently range of variation of $d$ and $q$ variables.

Figure 4 is examples of equivalent alternative configurations for the parallel robot 3RPS and 6SPS. 


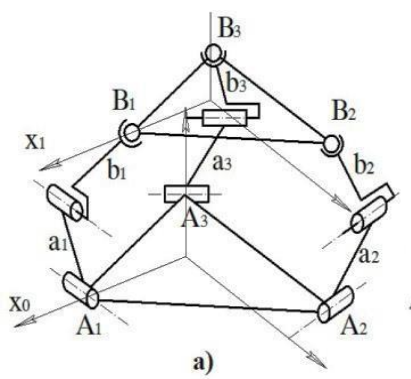

a)
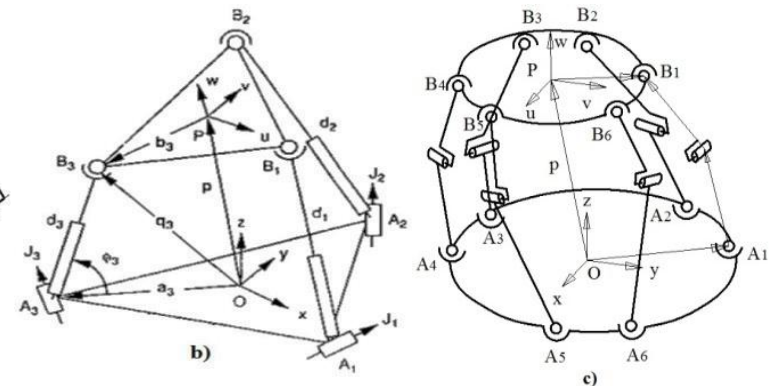

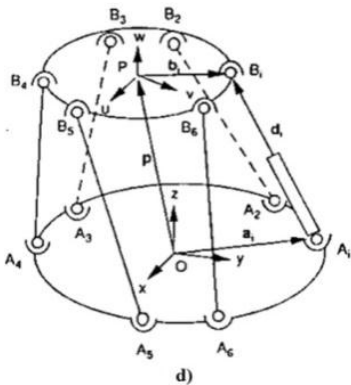

Figure 4. Original configurations of robot 3RPS, 6 SPS( 4b, 4d) and equivalent alternative configurations $(4 \mathrm{a}, 4 \mathrm{c})$ respectively

\subsubsection{In case of using two revolute joints to replace one prismatic}

In case the direction of prismatic joint axis is fixed as in robot 3PRRR (Figure 5a), the equivalent alternative configuration using two revolute joints RR to replace one prismatic joint $\mathrm{P}$ has constraints conditions attached as Figure $5 \mathrm{~b}$.

Because the direction of prismatic joint is fixed, only the distance between point $\mathrm{A}$ and point $\mathrm{C}$ in the direction of prismatic joint is changing. Hence, alternative configuration needs further description by other constraints conditions to create equivalency in terms of kinematics between the two configurations.

Consider alternative configuration of Figure 5b, to ensure that the movement of the prismatic joint is in accordance with the direction of $\mathrm{Y}$-axis, the additional mathematical constraints that the alternative configuration needs is:

$\mathrm{AB}=\mathrm{BC}=\mathrm{a}$ and $2 \mathrm{q}_{1}=\mathrm{q}_{2}$
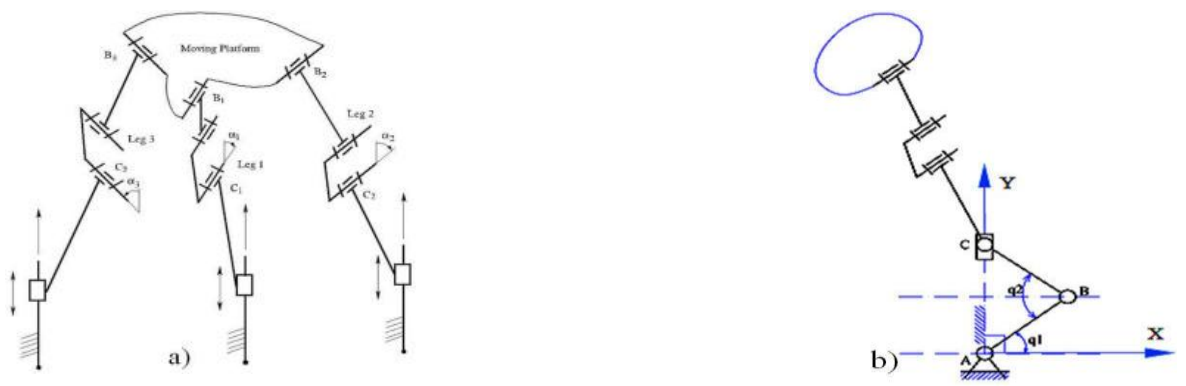

Figure 5. Robot 3 PRRR (5a) and equivalent alternative configuration (5b)

Verify the mathematical constraints, the trajectory equation of point $\mathrm{C}$ is in the following form:

$$
\left\{\begin{array}{l}
x_{c}=A B \cdot \cos \left(q_{1}\right)-B C \cdot \cos \left(\frac{q_{2}}{2}\right) \\
y_{c}=A B \cdot \sin \left(q_{1}\right)+B C \cdot \sin \left(\frac{q_{2}}{2}\right)
\end{array}\right.
$$

Substitute (9) to (10) has:

$$
\left\{\begin{array}{l}
x_{c}=0 \\
y_{c}=2 a \cdot \sin \left(q_{1}\right)
\end{array} \forall q_{1}\right.
$$

Thus with the constraint (9), the locus of point $\mathrm{C}$ is the line coincides with the vertical $\mathrm{Y}$-axis or is the movement direction of prismatic joint in the original configuration. 


\subsection{Form of objective function in equivalent alternative configuration}

After equivalent alternative configuration for each type of robot driven by prismatic joint has been identified, the next step needed to done is to develop the objective function of the alternative function. Displacement graph of the original configuration will completely be inferred from the displacement graph of alternative configuration based on geometric relations between the two configurations.

Suppose that in the original configuration objective function has the form:

$$
\left[T_{i}\left(d_{1}, d_{2} \ldots, d_{n}\right)^{2}-a_{i}^{2}\right]^{2}=0
$$

The change of variables formula of two configurations and mathematical constraints:

$$
d_{i}=f\left(\theta_{i}\right)
$$

The variation of the control variables in the two configurations is:

$$
d_{i \min } \leq d_{i} \leq d_{i \max } \Leftrightarrow \theta_{i \min } \leq \theta_{i} \leq \theta_{i \max }
$$

Objective function in alternative configuration has the following form:

$$
\left[T_{i}^{\prime}\left(\theta_{1}, \theta_{2} \ldots, \theta_{n}\right)-a_{i}^{\prime}\right]^{2}=0
$$

Equation (15) is always in the form of quadratic function and it is the equivalent alternative kinematic model.

\section{NUMERICAL SIMULATION EXAMPLE}

Consider control example of Stewart Gough robot (Figure 4d) following a trajectory in space (Figure 6), there is an equation:

$$
\left\{\begin{array}{l}
x= \pm \sqrt{10196 \cdot \sin ^{2}(t)-2187 \cdot \sin (t)-3898.828} \\
y= \pm 100 \cdot \cos (t) \\
z=100 \cdot \sin (t) \\
0.826 \leq t \leq 1.5708
\end{array}\right.
$$

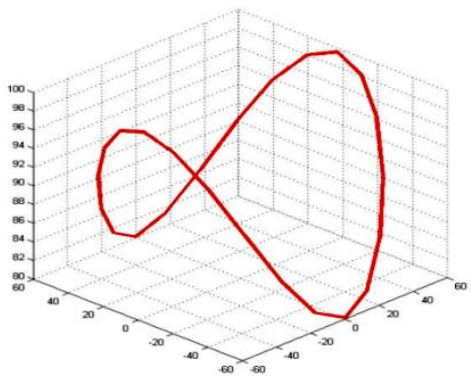

Figure 6. Trajectory of movable platen Stewart Gough robot that needs to control

Since prismatic joints axis of the Stewart Gough robot has direction that can be changed so the alternative configuration selected is the type in which a revolute joint replaces a prismatic joint Figure 7.

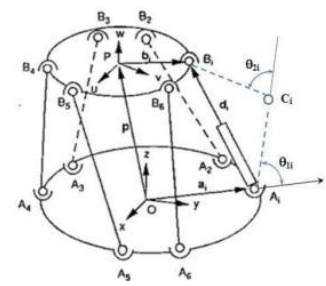

Figure 7. Geometric relation between original variable di and new variable $\theta_{2 i}$ 
Firstly, the change of variables formula must be developed. In the original configuration, control variable is the length of direction axis di, in the alternative configuration control variable is the angle $\hat{\theta}_{2 i}$.

To simplify, in alternative configuration suppose that the length of 2 links $A_{i} C_{i}=C_{i} B_{i}=a=$ const , then $\Delta A_{i} C_{i} B_{i}$ is isosceles triangle with apex $C_{i}$, apply the cosine function in $\Delta$ we have:

$$
\begin{aligned}
& A_{i} B_{i}^{2}=A_{i} C_{i}^{2}+C_{i} B_{i}^{2}-2 \times A_{i} C_{i} \times C_{i} B_{i} \times \cos \angle A_{i} C_{i} B_{i} \\
& \Leftrightarrow \angle A_{i} C_{i} B_{i}=\arccos \left(\frac{A_{i} C_{i}^{2}+C_{i} B_{i}^{2}-A_{i} B_{i}^{2}}{2 \times A_{i} C_{i} \times C_{i} B_{i}}\right)
\end{aligned}
$$

On the other hand $\hat{\theta}_{2 i}=180^{\circ}-\angle A_{i} C_{i} B_{i}$ so

$$
\hat{\theta}_{2 i}=180^{\circ}-\arccos \left(\frac{A_{i} C_{i}^{2}+C_{i} B_{i}^{2}-A_{i} B_{i}^{2}}{2 \times A_{i} C_{i} \times C_{i} B_{i}}\right) \Leftrightarrow \widehat{\theta}_{2 i}=180^{\circ}-\arccos \left(\frac{2 a^{2}-d^{2}}{2 a^{2}}\right)
$$

Equation (18) is the formula to change variables between two configurations.

Since the limbs of Stewart Gough robot have identical structures, detailed development of $i^{\text {th }}$ limb of robot is as shown in Figure 8.

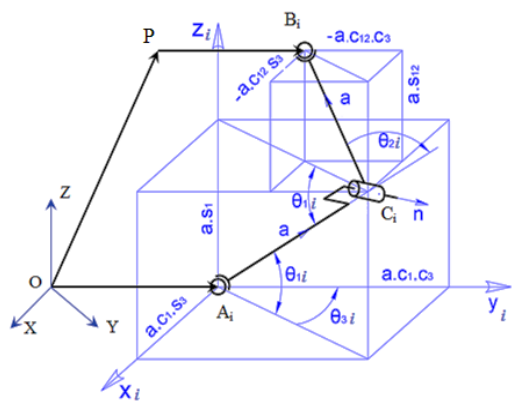

Figure 8. Detailed development diagram of $i^{\text {th }}$ limb of Stewart Gough robot in alternative configuration

Associated vector equation has the following form:

$$
\overrightarrow{O P}+R_{R P Y} \cdot \overrightarrow{P B_{i}}-\overrightarrow{O A}_{i}=\overrightarrow{A_{i} C_{i}}+\overrightarrow{C_{i} B_{i}} \Leftrightarrow \overrightarrow{O P}=\overrightarrow{A_{i} C_{i}}+\overrightarrow{C_{i} B_{i}}-R_{R P Y} \cdot \overrightarrow{P B_{i}}+\overrightarrow{O A} i
$$

where $R_{R P Y}$ is matrix Roll-Pitch-Yaw.

$$
R_{R P Y}=R(z, \gamma) R(y, \beta) R(x, \alpha)=\left[\begin{array}{ccc}
c \beta . c \gamma & s \alpha . s \beta . c \gamma-c \alpha . s \gamma & c \alpha . s \beta . c \gamma+s \alpha . s \gamma \\
c \beta . s \gamma & s \alpha . s \beta . s \gamma+c \alpha . c \gamma & c \alpha . s \beta . s \gamma-s \alpha . c \gamma \\
-s \beta & s \alpha . c \beta & c \alpha . c \beta
\end{array}\right]
$$

Detailed development (19):

$$
\left[\begin{array}{c}
p_{x} \\
p_{y} \\
p_{z}
\end{array}\right]=\left[\begin{array}{c}
a \cdot s \theta_{3 i} \cdot c \theta_{1 i}+a \cdot s \theta_{3 i} \cdot c\left(\theta_{1 i}+\theta_{2 i}\right) \\
a \cdot c \theta_{3 i}+a \cdot \cos \left(\theta_{2 i}\right) \cdot \cos \left(\theta_{3 i}\right) \\
a \cdot s \theta_{3 i} \cdot s \theta_{1 i}+a \cdot s \theta_{3 i} \cdot s\left(\theta_{1 i}+\theta_{2 i}\right)
\end{array}\right]+\left[\begin{array}{c}
x_{A i} \\
y_{A i} \\
z_{A i}
\end{array}\right]-\left[\begin{array}{ccc}
c \beta . c \gamma & s \alpha \cdot s \beta \cdot c \gamma-c \alpha \cdot s \gamma & c \alpha . s \beta \cdot c \gamma+s \alpha \cdot s \gamma \\
c \beta . s \gamma & s \alpha \cdot s \beta \cdot s \gamma+c \alpha \cdot c \gamma & c \alpha \cdot s \beta \cdot s \gamma-s \alpha \cdot c \gamma \\
-s \beta & s \alpha \cdot c \beta & c \alpha \cdot c \beta
\end{array}\right] \cdot\left[\begin{array}{c}
x_{c i} \\
y_{c i} \\
z_{c i}
\end{array}\right]
$$


where " $c$ " and " $s$ " are symbols of cosine and $\sin ; \alpha, \beta, \gamma$ are the orientation angle of moving platform. When the objective function is structured based on (2), objective function of robot is determined as follows:

$$
\begin{aligned}
& \left\{\left[p_{x}-a . s \theta_{3 i} . c \theta_{1 i}-a . s \theta_{3 i} . c\left(\theta_{1 i}+\theta_{2 i}\right)+c \beta . c \gamma \cdot x_{C i}+(s \alpha . s \beta . c \gamma-c \alpha . s \gamma) \cdot y_{C i}+\right.\right.
\end{aligned}
$$

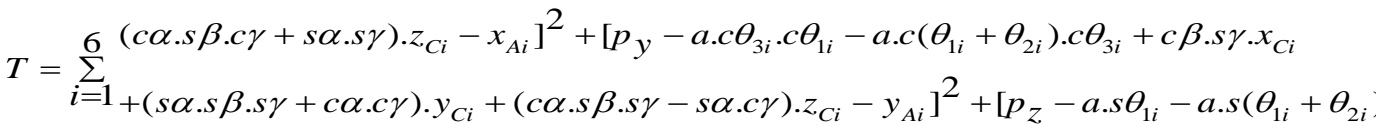

$$
\begin{aligned}
& \left.\left.-s \beta \cdot x_{C i}+s \alpha \cdot c \beta \cdot y_{C i}+c \alpha \cdot c \beta \cdot z_{C i}-z_{A i}\right]^{2}\right\} \Rightarrow \text { minimize }
\end{aligned}
$$

Using the GRG algorithm to solve equation (22), the results are displacement graph of 6 control joints $\theta_{2 i}$ passing through 24 key points of the trajectory in alternative configuration and converted displacement graph of prismatic joint di in original configuration as in Figure 9.
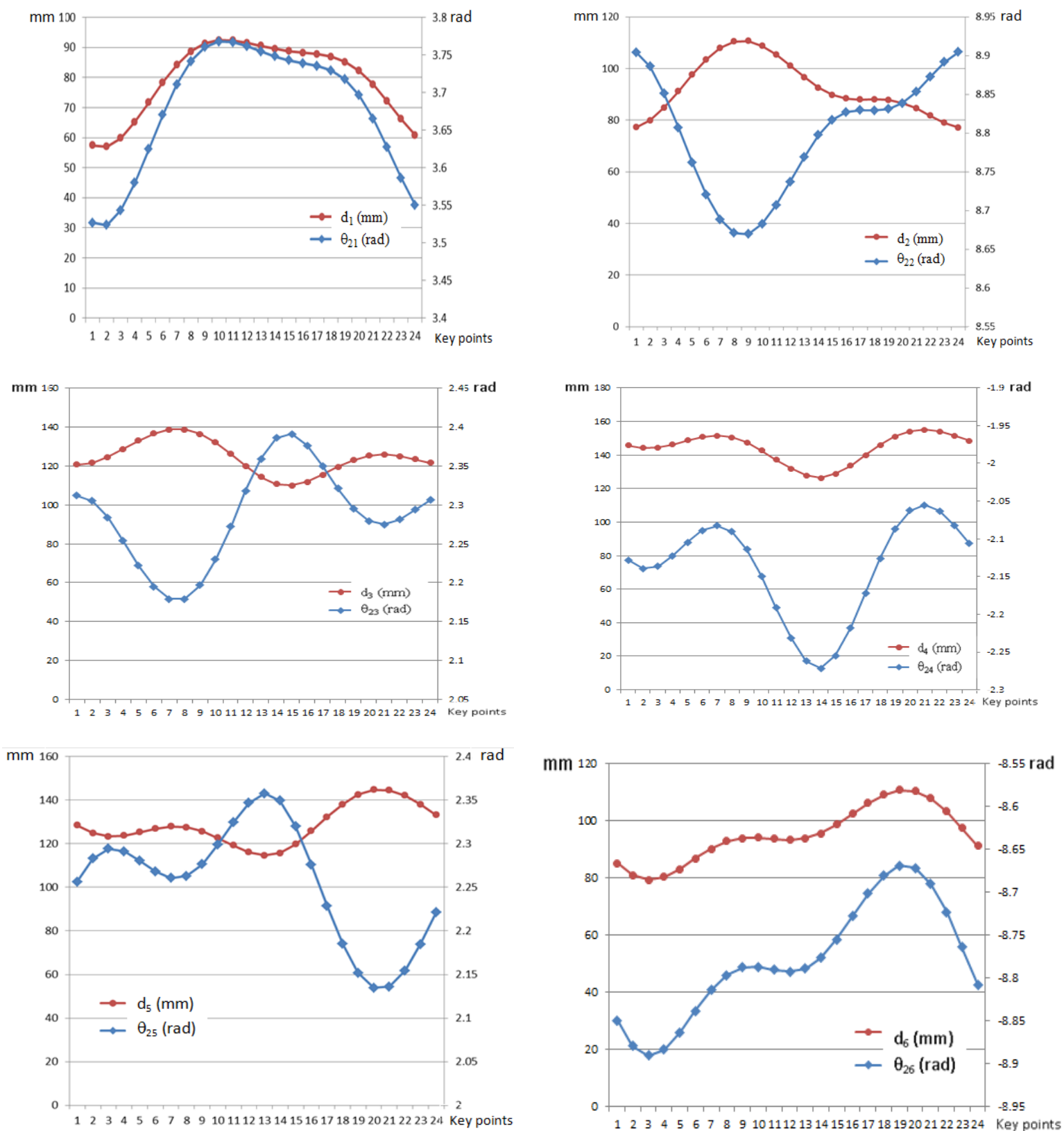

Figure 9. The converted displacement graph of 6 equivalent joint variables between the replacement configuration and the original structure

A Techniques to Downgrade Downgrade Objective Function in Parallel Robot ... (Trang Thanh Trung) 
By using alternate configuration to find displacement graph of 6 limbs use revolute joint, use the formula changed to redefine the displacement graph of 6 limbs use prismatic joint in the original configuration. This is the kinematics control information of Stewart Gough robot.

\section{CONCLUSION}

By using the technique to downgrade objective function based on equivalent alternative configuration, the difficulties encountered regarding the order of the objective function forms solving parallel robot kinematics problems has been resolved. Equivalent alternative configurations proposed in the article use one revolute joint or a combination of two revolute joints to replace a prismatic joint along with different kinematic constraints described in solution selection conditions of optimization problem. All parallel robots having the form of quaternary objective function can be downgraded to quadratic objective function, from which the parallel robot kinematics problem can be simplified and its solutions can be quickly found. This technique has been tested on many different types of parallel robot with different structures and the results show that the applicability of this technique in practice is very high.

Parallel robot kinematics problem often has many mathematical solutions, in addition to finding accurate control solution, identification of extra parameters $\left(\hat{\theta}_{1 i}, \hat{\theta}_{3 i}\right.$ as in example of section 5) in limbs of parallel robot to establish a unique solution relation in joint space and workspace is an issue needed discussed. This is a research direction that we will discuss in another paper.

\section{REFERENCES}

[1] Y.D. Patel, P.M. George, Parallel manipulators applications-A survey. Modern Mechanical Engineering, vol. 2, pp. 57-64, 2012.

[2] Tanio K.Tanev. Kinematics of a hybrid (parallel-serial) robot manipulator. Mechanism and Machine Theory, vol 35(9), pp. 1183-1196, September 2000.

[3] Serdar Kucuk. Kinematics, Singularity and Dexterity Analysis of Planar Parallel Manipulators Based on DH Method. Robot Manipulators New Achievements, Aleksandar Lazinica and Hiroyuki Kawai (Ed.). InTech, pp. 387400, 01 April, 2010.

[4] Kang, B.; Chu, J. \& Mills, J. K. Design of high speed planar parallel manipulator and multiple simultaneous specification control. Proceedings of IEEE International Conference on Robotics and Automation, South Korea, pp. 2723-2728, 2001.

[5] Luc Rolland, The Forward Kinematics Problem with an exact algebraic Method for the general parallel robot. VSP and Robotics Society of Japan, vol 19( 9), pp. 995- 1025, 2005.

[6] Sapra. R, Mathew. M, Majumder. S. A Solution to Inverse Kinematics Problem Using the Concept of Sampling Importance Resampling. Advanced Computing \& Communication Technologies (ACCT), Fourth International Conference on, Rohtak, pp. 471 - 477, 8-9 Feb. 2014

[7] J.-P. MERLET. Parallel Robots (Second Edition). Published by Springer, P.O. Box 17, 3300 AA Dordrecht, The Netherlands 2006.

[8] Ozgur Yeniay. A comparative study on optimization methods for the constrained nonlinear programming problems. Mathematical Problems in Engineering, Hindawi Publishing Corporation, vol 2, pp.165-173, 2005.

[9] Pham Thanh Long, A New Method to Solve the Reverse Kinematic Robot Problem. ISTS Swissotel Le Concorde, Bangkok Thailand, pp. 43-46, November 21-24/2012

[10] Domagoj Jagobovic, Ocjena ucinkovitosti postupaka za rjesavanje kinematike Stewartowih paralelnih mehanizama. Magistarski Rad, zagreb, pp. 81, 2001.

[11] L. Yan and D. Ma. Global Optimization for constrained nonlinear programs using line-up competition algorithm. Compus. Oper. Res, vol 25(11-22), pp. 1601-1610, 2001.

[12] L. S. Lasdon, A. D. Warren, A. Jain, and M. Ratner (1978) Design and Testing of a generalized reduced gradient code for nonlinear Programming, ACM Trans. Math. SoftWare, vol 4(1), pp. 34-50, 1978.

\section{BIOGRAPHIES OF AUTHORS}

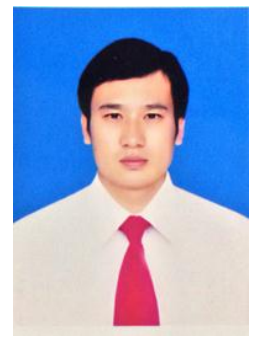

Trang Thanh Trung was born in Gang Thep, Thai Nguyen City, Viet Nam in 1983. He is the Ph. D. candidate at the School of Mechanical and Automotive Engineering, South China University of Technology, China. He received the B.S, M.S. degrees in Mechanical Engineering from Thai Nguyen University, Viet Nam in 2006, 2010, respectively.

From 2008 to 2012, he was a lecturer at Thai Nguyen University of Technology, Viet Nam. His research interest covers robot control technology and machine learning. There were 02 articles published in magazines and conferences. Corresponding author of this paper. 


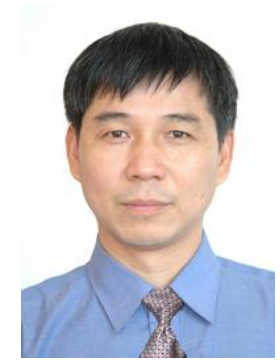

Li Wei Guang (1958, Male), PhD, Professor and doctoral supervisor. He received the B.S, M.S. and Ph.D. degree in mechanical engineering from the Mechanical and Automotive Engineering, South China University of Technology, China in 1982, 1996, 1999, respectively.

From 2001 to 2015, he was a director of the research of modern NC Technology, School of mechanical and automotive engineering. South China University of Technology. Vice President of Guangdong manufacturing information society, director of hardware products Standardization Technical Committee. His research interest covers modern $\mathrm{CNC}$ equipment, manufacturing system of digital and information control, industrial robot technology, electromechanical integration equipment, steam turbine bearing, and the shafting vibration monitoring, and vibration reduction technology.

He has been more than 10 technical achievements in enterprise application and industrialization of new technologies for $\mathrm{CNC}$ machining center is to promote. Optical-mechanical-electrical integration results of the aviation industry, scientific and technological progress Award 1995, CNC technology, Guangdong Provincial Science and technology progress award of Guangdong excellent new product Award. He has won more than 30 national invention patent and utility model patents. In recent years, he has more than 100 papers published in national and international academic papers, editors published a modern manufacturing technology (mechanical industry publishing house), mechanical control Foundation (bilingual tutorial) (Wuhan University of technology press), the machinery and equipment of CNC technology (national defense industry press) and 3 textbooks.

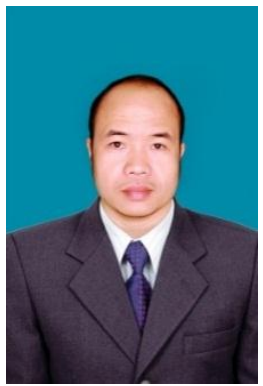

Pham Thanh Long was born in Vinh Phu, Viet Nam in 1977. He received the B.S, M.S. and $\mathrm{Ph} . \mathrm{D}$. degree in mechanical engineering from Faculty of Mechanical Engineering, Thai Nguyen University of Technology, Viet Nam in 1995, 2001, 2006, respectively.

From 2000 to 2008, he was a lecturer at Faculty of Mechanical Engineering, Thai Nguyen University of Technology, Viet Nam. From 2008 to 2015, he was the head of Department of Mechatronics, Thai Nguyen University of Technology. His research interest covers mechatronics, robotics, automation in manufacturing, CAD/CAM/CNC technology. He has more than 17 papers published in national and international academic papers, 03 textbooks, and participated in 7 research topics in Vietnam. 